\title{
Worldwide Incidence of Colorectal Cancer, Leukemia, and Lymphoma in Inflammatory Bowel Disease: An Updated Systematic Review and Meta-Analysis
}

\author{
Chelle L. Wheat, ${ }^{1,2}$ Kindra Clark-Snustad, ${ }^{2}$ Beth Devine, ${ }^{1,3}$ David Grembowski, \\ Timothy A. Thornton, ${ }^{4}$ and Cynthia W. Ko ${ }^{2}$ \\ ${ }^{1}$ School of Public Health, Department of Health Services, University of Washington, Seattle, WA 98195, USA \\ ${ }^{2}$ Division of Gastroenterology, Department of Medicine, University of Washington, Seattle, WA 98195, USA \\ ${ }^{3}$ School of Pharmacy, Department of Pharmacy, University of Washington, Seattle, WA 98195, USA \\ ${ }^{4}$ School of Public Health, Department of Biostatistics, University of Washington, Seattle, WA 98195, USA \\ Correspondence should be addressed to Chelle L. Wheat; wheatc@uw.edu
}

Received 8 February 2016; Accepted 21 March 2016

Academic Editor: Bjørn Moum

Copyright ( 2016 Chelle L. Wheat et al. This is an open access article distributed under the Creative Commons Attribution License, which permits unrestricted use, distribution, and reproduction in any medium, provided the original work is properly cited.

\begin{abstract}
Background/Aims. Inflammatory bowel disease (IBD) is associated with an increased risk of colorectal cancer (CRC). In addition, there may be an association between leukemia and lymphoma and IBD. We conducted a systematic review and meta-analysis of the IBD literature to estimate the incidence of CRC, leukemia, and lymphoma in adult IBD patients. Methods. Studies were identified by a literature search of PubMed, Cochrane Library, Medline, Web of Science, Scopus, EMBASE, and ProQuest Dissertations and Theses. Pooled incidence rates (per 100,000 person-years [py]) were calculated through use of a random effects model, unless substantial heterogeneity prevented pooling of estimates. Several stratified analyses and metaregression were performed to explore potential study heterogeneity and bias. Results. Thirty-six articles fulfilled the inclusion criteria. For CRC, the pooled incidence rate in CD was 53.3/100,000 py (95\% CI 46.3-60.3/100,000). The incidence of leukemia was $1.5 / 100,000$ py $(95 \%$ CI $-0.06-3.0 / 100,000)$ in IBD, $0.3 / 100,000$ py $(95 \%$ CI $-1.0-1.6 / 100,000)$ in CD, and $13.0 / 100,000$ py $(95 \%$ CI $5.8-20.3 / 100,000)$ in UC. For lymphoma, the pooled incidence rate in CD was $0.8 / 100,000$ py $(95 \%$ CI $-0.4-2.1 / 100,000)$. Substantial heterogeneity prevented the pooling of other incidence estimates. Conclusion. The incidence of CRC, leukemia, and lymphoma in IBD is low.
\end{abstract}

\section{Introduction}

Colorectal cancer (CRC) incidence is higher in inflammatory bowel disease (IBD) patients than in the general population, and CRC accounts for an estimated $10-15 \%$ of deaths in patients with IBD [1]. The risk conferred by IBD may be due to chronic inflammation combined with genetic factors [13]. Patients with extensive inflammation, a younger age at diagnosis, long disease duration, comorbid primary sclerosing cholangitis (PSC), and pseudopolyposis are at the highest risk [4-14].

IBD patients receiving immunomodulators may or may not also be at higher risk of lymphoproliferative disorders such as lymphoma and leukemia [15-19]. The risk of lymphoma in IBD patients is low but appears to be higher than in the general population $[6,8,14,20-22]$. The risk of leukemia in IBD is less clear $[6,8,14,23,24]$.

Understanding the risk of development of these malignancies inherent to IBD is crucial for cancer surveillance strategies. In addition, determination of the absolute increase in risk of these malignancies from IBD pharmacotherapy is a crucial consideration for providers and patients. The aims of this study are to estimate the incidence of CRC, leukemia, and lymphoma in adult IBD patients through a systematic review and meta-analysis. Unique to this study, we attempt to evaluate the underlying risk of these cancers in IBD overall and separately Crohn's Disease (CD) and ulcerative colitis (UC) and exclude the effects of IBD pharmacotherapy (specifically immunomodulators and biologics), given the evidence that these medications may increase cancer risk. 


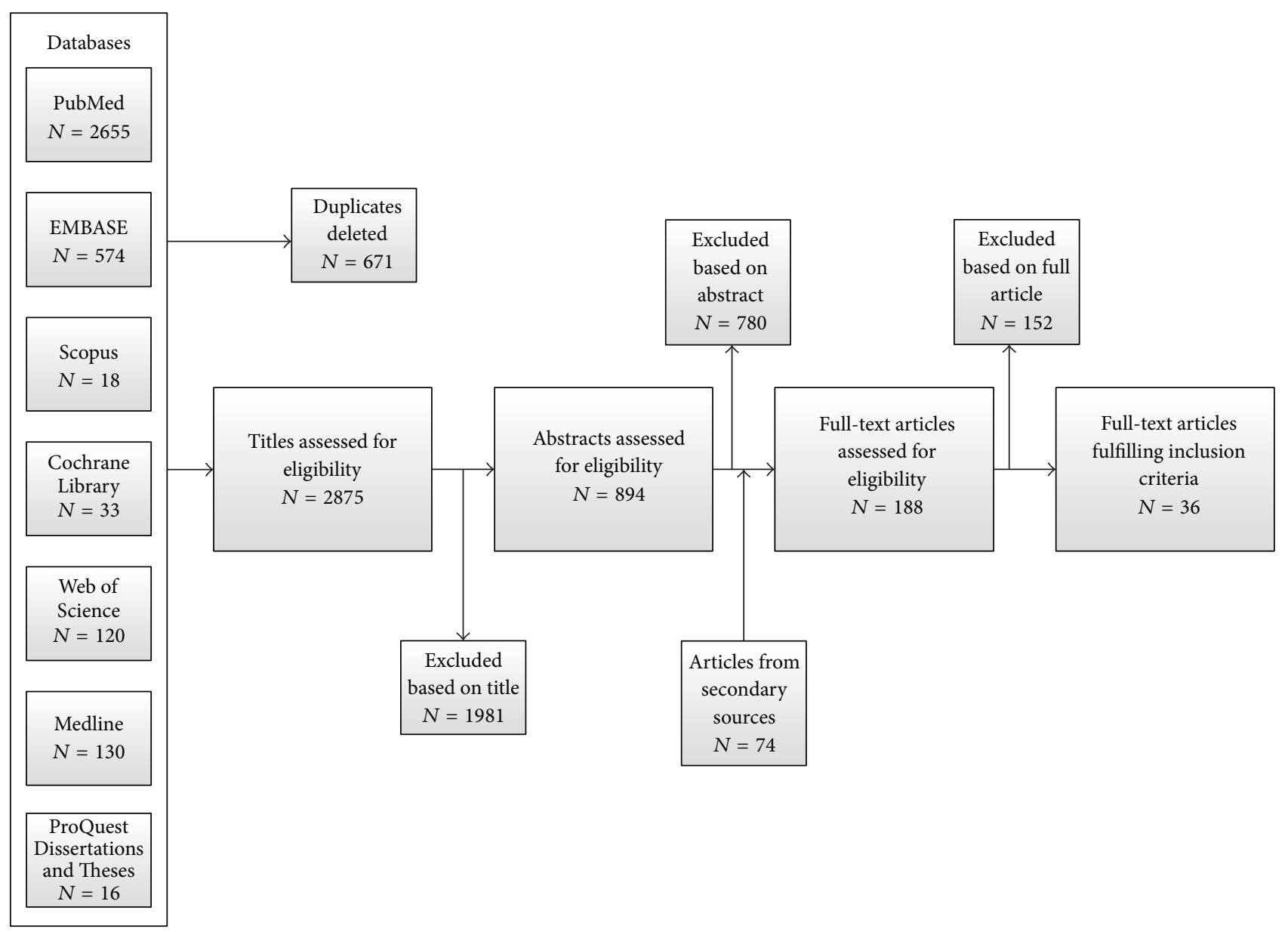

Figure 1: Flowchart depicting the identification of studies, inclusion, and exclusion assessment.

\section{Materials and Methods}

2.1. Literature Search. A detailed literature search was conducted to identify all published and unpublished studies examining the incidence of CRC, leukemia, and lymphoma in adult IBD patients. We searched the PubMed, Cochrane Library, Medline, Web of Science, Scopus, EMBASE, and ProQuest Dissertations and Theses databases. Reference lists of published articles were hand searched for secondary sources and experts in the field contacted for unpublished data. Furthermore, https://clinicaltrials.gov/, the WHO International Clinical Trial Registry, and scientific information packets of approved IBD pharmacotherapies were scrutinized for additional information sources. No restrictions on language, country of origin, or publication date were used. Figure 1 outlines the literature search and Supplementary Table 1 (in Supplementary Material available online at http://dx.doi.org/10.1155/2016/1632439) details the search strategy employed.

2.2. Inclusion and Exclusion Criteria. All studies that reported incidence or provided information sufficient to accurately calculate incidence for the three cancers of interest in adult IBD patients were included. Studies focusing on pediatric populations, not reporting person-years of follow-up, of duration less than one year, and not written in English and unable to be translated to English were excluded. If publications reported duplicate data on a population, only the publication with the longest follow-up period was included.

2.3. Data Collection and Quality Assessment. Two independent reviewers (CW and KCS) examined each article for inclusion according to the eligibility criteria. Any disagreement was resolved through discussion and consensus. Thirtysix articles fulfilled the inclusion criteria. Twenty-five articles reported incidence estimates for CRC $[7,10,11,13,14,18,19$, $21,22,24-39]$, ten for leukemia $[8,14,18,19,21-24,33,39]$, and twenty-one for lymphoma $[8,10,14,18,19,21,22,24$, $26,33,34,39-48$ ] (some articles reported incidence estimates for multiple cancers). Figure 1 outlines the search flowchart.

We retrieved demographic (where possible) and outcome data for each included article using standardized forms. Individual studies were assigned a bias risk rating using the Cochrane Collaboration's Risk of Bias Assessment Tool: 
for Non-Randomized Studies of Interventions (ACROBATNRSI) [49]. The strength of evidence for each cancer was assessed utilizing the Grades of Recommendation, Assessment, Development, and Evaluation (GRADE) approach [50].

2.4. Statistical Analysis. Individual study unadjusted incidence rates (per 100,000 person-years [py]) were calculated from the reported number of cancer cases and person-years of follow-up for each outcome separately. Standard errors and 95\% confidence intervals (CIs) were estimated assuming a Poisson distribution [51]. In situations with zero observed cases, the value of 3.7 was used to calculate incidence rates and the confidence interval upper limit [51].

As our interest is in quantifying the incidence rate of CRC, leukemia, and lymphoma in IBD patients not treated with immunomodulators or biologic agents (and treatment information is often unreported), two stratification variables were created using study publication year as an estimate of when each medication class became widely used. 1995 was used as the dividing year for widespread immunomodulator use and 2000 for biologic use. Pooled incidence rates with 95\% CIs were then calculated for (1) each cancer overall, (2) each cancer in CD and UC separately, (3) each cancer stratified by year of publication, and (4) each cancer stratified by country of origin (to determine if incidence varied by geographic region). A random effects model was used to account for potential between-study variations. The $I^{2}$ statistic was used to quantify the percentage of heterogeneity for all pooled estimates from between-study variation, with $\geq 75 \%$ indicating substantial heterogeneity [52]. Publication bias and the presence of other small study effects were measured through visual assessment of funnel plot symmetry and Egger's test [52]. Sensitivity analyses were undertaken to explore potential sources of heterogeneity. Metaregression was used to further test the effects of study- and subject-level covariates on cancer risk, as well as the degree of between-study heterogeneity explained by the covariates through calculation of the adjusted $R^{2}$. The adjusted $R^{2}$ measures the relative reduction in the between-study variance explained by the covariates in the model and is presented as a percentage [52]. Statistical analysis was performed using Stata (StataCorp, College Station, TX). $p$ values $\leq 0.05$ were considered statistically significant.

\section{Results and Discussion}

\subsection{Results}

3.1.1. Colorectal Cancer. Reported incidence rates of CRC in IBD ranged from $41.5 / 100,000$ py (95\% CI $24.5-58.5 / 100,000)$ to $543.5 / 100,000$ py (95\% CI 316.4-770.6/100,000) (Table 1). Substantial heterogeneity prevented pooling of estimates using a random effects model (heterogeneity test, $\mathrm{chi}^{2}=$ 174.65; $\left.p<0.001 ; I^{2}=86.3 \%\right)$. Therefore, we present unpooled incidence estimates. Separate sensitivity analyses excluding the studies with the highest individual incidence estimate [31] and the study with the greatest weight on the pooled estimate [7] did not significantly change the degree of heterogeneity present.

Reported CRC incidence rates in CD ranged from 19.5/ $100,000$ py (95\% CI $0.4-38.6 / 100,000)$ to $344.9 / 100,000$ py (95\% CI 105.9-583.9/100,000) (Table 1). Using a random effects model, an estimated incidence of CRC in CD of $53.3 / 100,000$ py (95\% CI 46.3-60.3/100,000) was obtained. Figure 2 displays the Forest plot for the pooled estimates. In $\mathrm{UC}$, the reported incidence rates ranged from $54.5 / 100,000$ py (95\% CI $30.0-79.0 / 100,000)$ to $543.5 / 100,000$ py (95\% CI 316.4-770.6/100,000). Substantial heterogeneity was again present when pooling using a random effects model (heterogeneity test, $\mathrm{chi}^{2}=110.7 ; p<0.001 ; I^{2}=86.4 \%$ ), and thus the results in UC were not pooled.

Analyses stratified by publication year and region of origin did not reveal any significant differences in results. We also conducted metaregression analyses to evaluate the potential impact of age, gender, race, Montreal Classification, disease duration, surgical history, smoking status, comorbid primary sclerosing cholangitis, presence of extraintestinal manifestations, and concomitant treatment with immunosuppressants and/or biologics on the CRC incidence in IBD. Due to the limited sample size and incomplete reporting of demographic characteristics in many studies, these analyses were underpowered. Together, age, gender, and disease duration explained a significant proportion of the betweenstudy variability (adjusted $R^{2}=65.67 \%$ ); however we could not make any further conclusions regarding the impact of these covariates on CRC incidence in IBD. Evaluation of funnel plots and Egger's test showed evidence of small study effects and/or publication bias for IBD overall $(p=0.149)$ and weak evidence of small study effects in CD and UC ( $p=0.005 \mathrm{CD} ; p=0.05 \mathrm{UC}$ ). However, the power of these tests may be compromised due to small sample sizes and significant heterogeneity between studies. Given the observational nature of the included studies and the probability of bias from small study effects, the overall quality of the CRC body of evidence per the GRADE approach is low.

3.1.2. Leukemia. Reported incidence rates of leukemia in IBD ranged from $0.0 / 100,000$ py $(95 \%$ CI $0.0-3.7 / 100,000)$ to $28.4 / 100,000$ py $(95 \%$ CI $-3.7-60.5 / 100,000)$ (Table 2). Using a random effects model, the pooled estimated incidence of leukemia in IBD of $1.5 / 100,000$ py was obtained (95\% CI $-0.02-3.0 / 100,000)$. Figure 3 illustrates the Forest plot for the pooled estimates. Moderate between-study heterogeneity was seen (heterogeneity test $\mathrm{chi}^{2}=23.8, p=0.005 ; I^{2}=62.1 \%$ ); however this is likely influenced by the small number of available studies. In CD, the range of reported incidence rates was identical to that of IBD (Table 2). In UC, reported incidence rates ranged from $8.97 / 100,000$ py $(95 \%$ CI $0.2-17.8 / 100,000)$ to $25.4 / 100,000$ py $(95 \% \mathrm{CI}-9.8-60.6 / 100,000)$ (Table 2$)$. The pooled incidence estimate was $0.3 / 100,000$ py for CD $(95 \%$ CI $-1.0-1.6 / 100,000)$ and $13.0 / 100,000$ py for UC (95\% CI 5.8$20.3 / 100,000)$. The $I^{2}$ statistics are $44.3 \%$ (heterogeneity test, $\mathrm{chi}^{2}=10.8, p=0.096$ ) and $0.0 \%$ (heterogeneity test, $\mathrm{chi}^{2}$ $=2.65, p=0.449$ ), respectively, indicating low levels of heterogeneity; however the power of this analysis is severely limited due to the small number of included studies. 


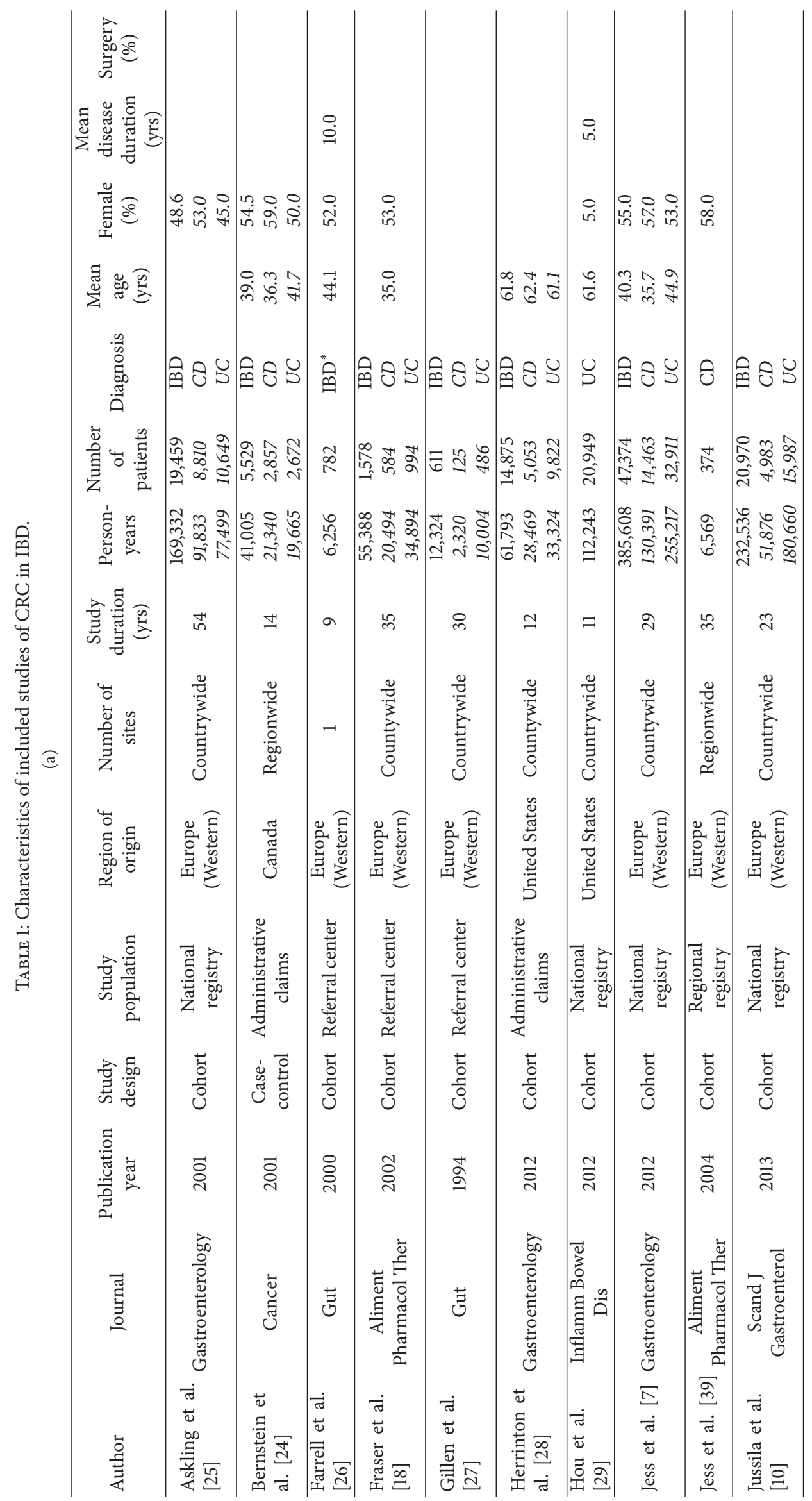




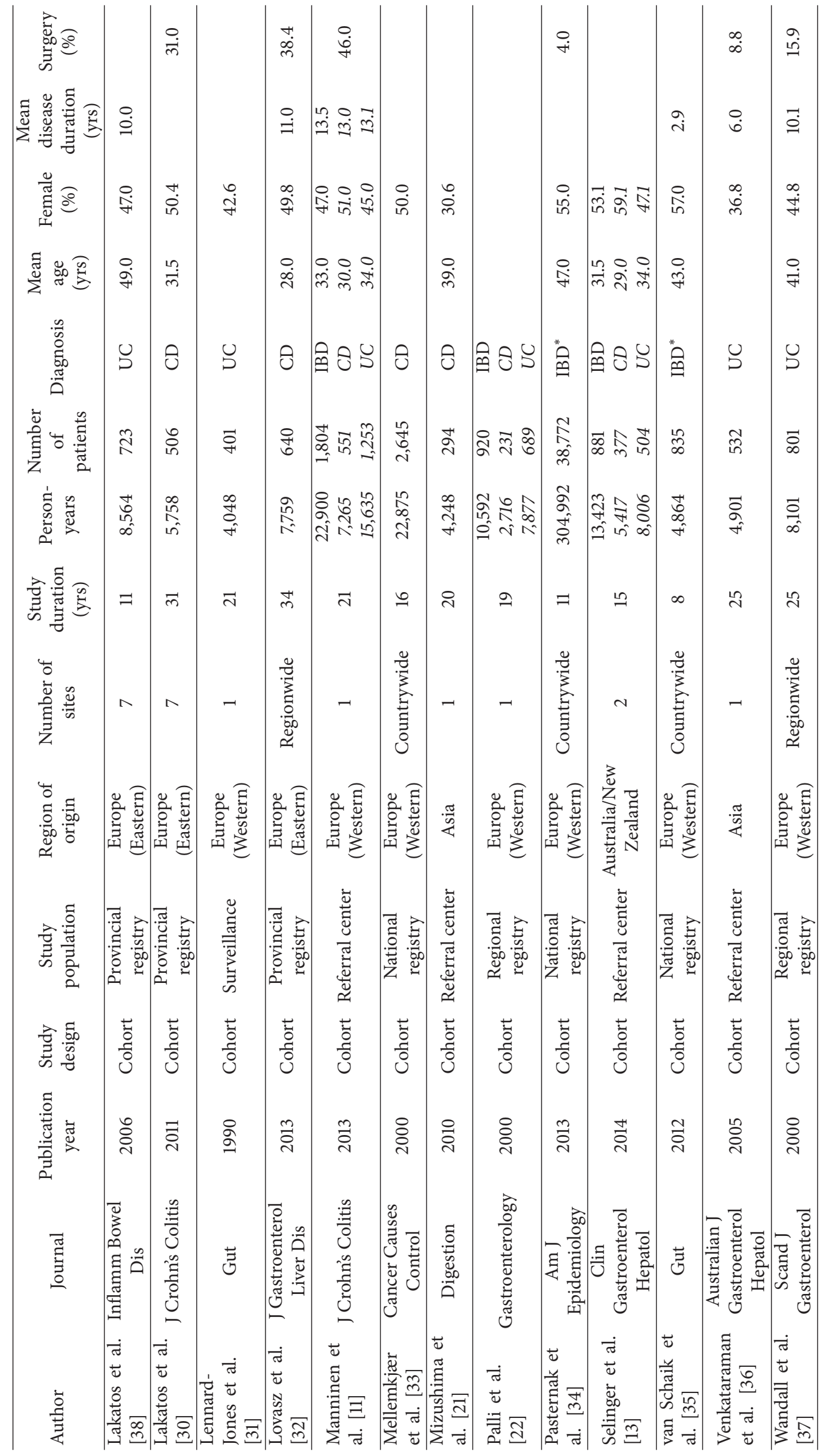




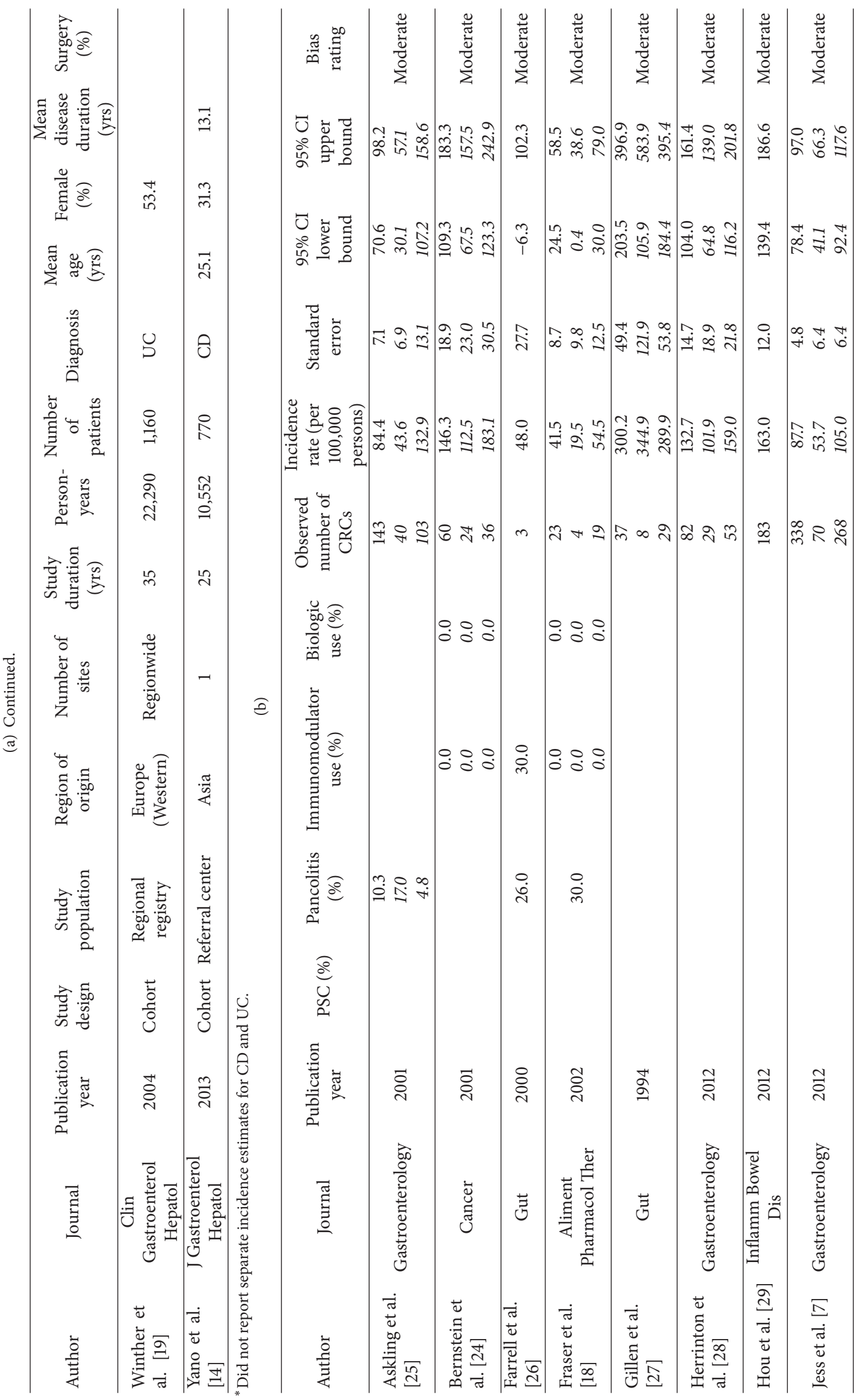




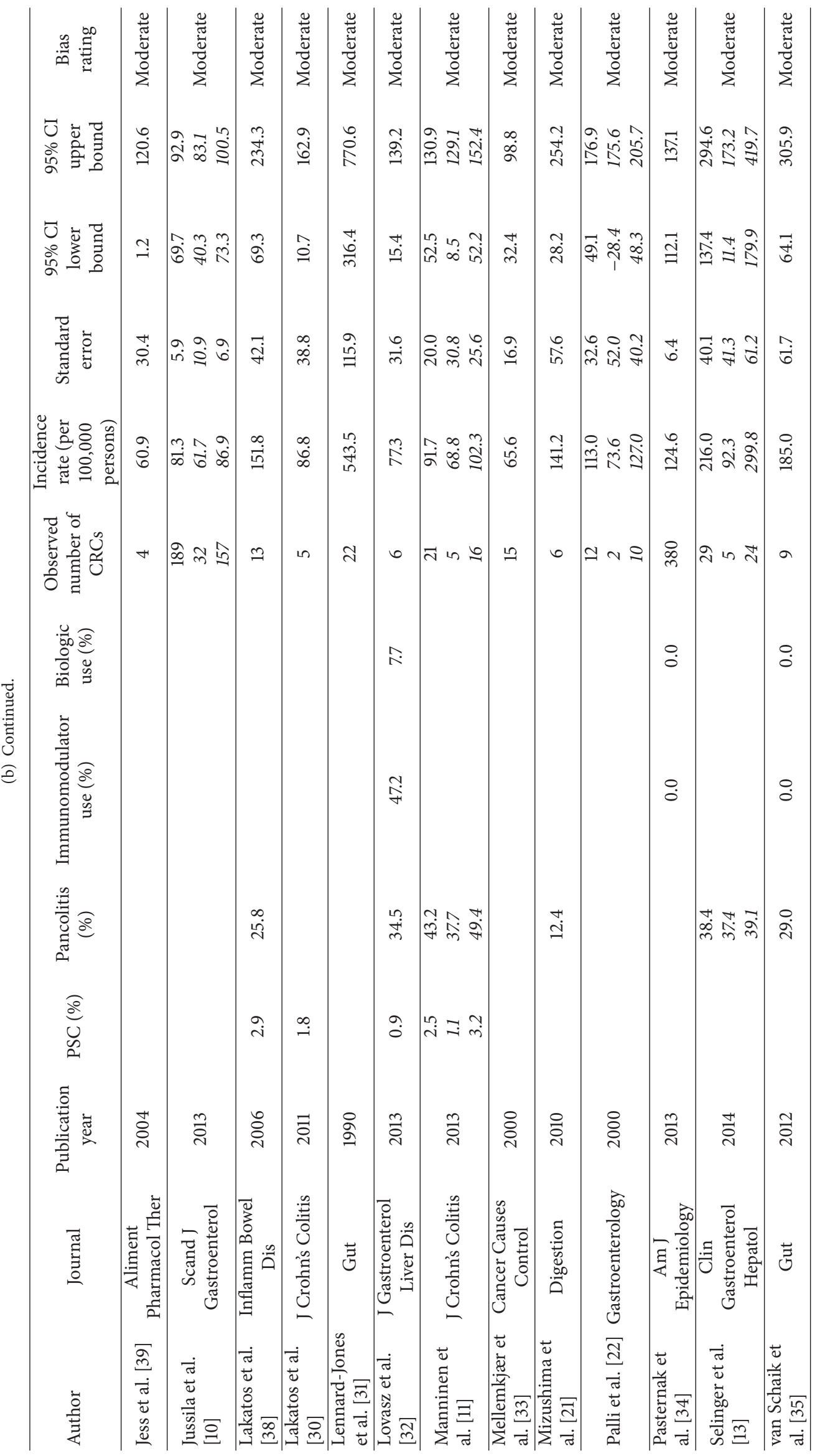




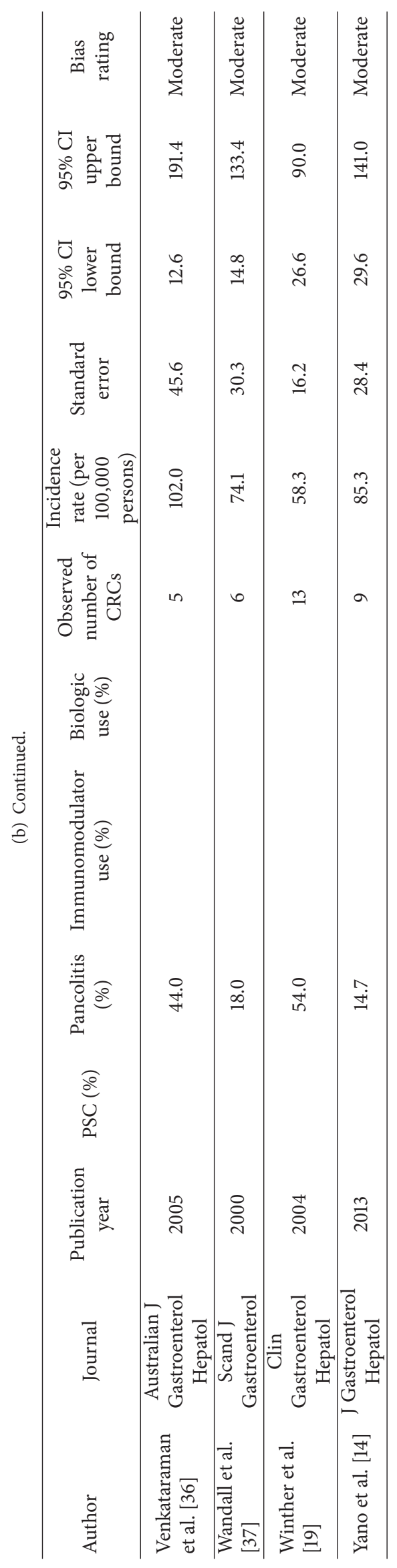




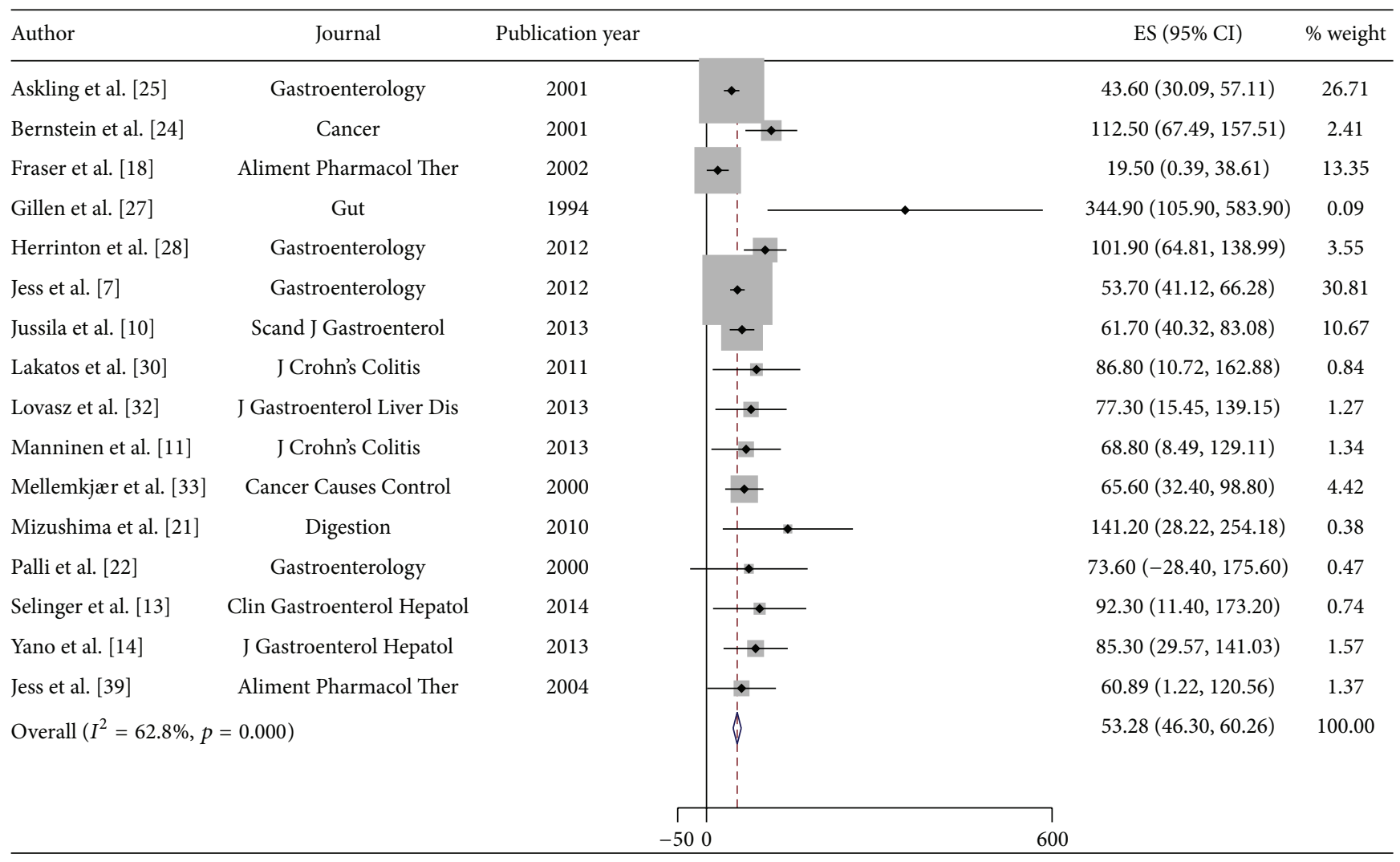

FIGURE 2: Incidence of colorectal cancer (CRC) in patients with Crohn's Disease (CD). Each incidence estimate is presented followed by the $95 \%$ confidence intervals (CIs). Each square in the plot indicates the point estimate of the incidence. The diamond represents the summary incidence from the pooled studies. Error bars depict the 95\% CIs.

Stratification by publication year and region did not impact the incidence estimates for IBD or for CD and UC separately. Furthermore, no significant effects of any study- or subject-level covariates on incidence estimates were discovered in metaregression analyses; however the small sample size again restricted the power of these tests.

As less than 10 studies were included, the interpretation of funnel plot symmetry and Egger's test to assess the presence of small study effects and/or publication bias are not recommended [52]. The overall quality of the leukemia body of evidence, per the GRADE approach, is low due to study designs and small sample size.

3.1.3. Lymphoma. Reported incidence rates for lymphoma in IBD ranged from $0.0 / 100,000$ py $(95 \%$ CI $0.0-3.7 / 100,000)$ to $81.7 / 100,000$ py $(95 \%$ CI $21.2-142.2 / 100,000)$ (Table 3 ). Substantial heterogeneity between studies prevented pooling of estimates (heterogeneity test, $\mathrm{chi}^{2}=591.1 ; p<0.001 ; I^{2}=$ $96.6 \%)$. Thus, the included studies are presented as unpooled estimates. A sensitivity analysis excluding the two studies with the lowest individual incidence estimates and highest weights on the pooled estimates was conducted, with no significant corresponding decrease in heterogeneity [14, 21].

Reported incidence rates of lymphoma in $\mathrm{CD}$ ranged from $0.0 / 100,000$ py $(95 \%$ CI $0.0-3.7 / 100,000)$ to $62.2 /$ 100,000 py (95\% CI 16.1-108.3/100,000) (Table 3). For UC, the incidence rates ranged from $0.0 / 100,000$ py $(95 \%$ CI $0.0-$ $3.7 / 100,000)$ to $76.2 / 100,000$ py $(95 \%$ CI $15.2-137.2 / 100,000)$ (Table 3). A pooled incidence rate of $0.6 / 100,000$ py ( $95 \%$ CI $-0.5-1.6 / 100,000)$ for CD was obtained. Substantial heterogeneity prevented pooling of estimates for UC (heterogeneity test, chi $\left.^{2}=199.5 ; p<0.001 ; I^{2}=94.5 \%\right)$. A sensitivity analysis excluding the study with the largest impact on the pooled estimate in UC [47] decreased the heterogeneity (heterogeneity test, $\mathrm{chi}^{2}=44.79 ; p<0.001 ; I^{2}=77.7 \%$ ). However, substantial heterogeneity remained, and results for UC are presented as unpooled estimates (Figure 4).

Incidence estimates stratified by publication year and region did not differ. Metaregression analysis revealed a statistically significant effect of age on lymphoma incidence in IBD. For each mean year increase in age, the incidence of lymphoma increased by approximately $2.1 / 100,000$ py ( $95 \%$ CI $0.74-3.4 / 100,000$ ), explaining approximately $65.8 \%$ of the between-study heterogeneity (adjusted $R^{2}=65.8 \%$ ). No other covariate effects were found in metaregression analyses.

There was weak evidence of publication bias and/or small study effects in the IBD analysis $(p=0.213)$ and in the UC analysis $(p=0.824)$. The number of included studies for CD is less than 10; thus analyses of funnel plots and Egger's test are not recommended [52]. The overall quality of the lymphoma body of evidence, per the GRADE approach, is low due to the observational designs of available studies. 


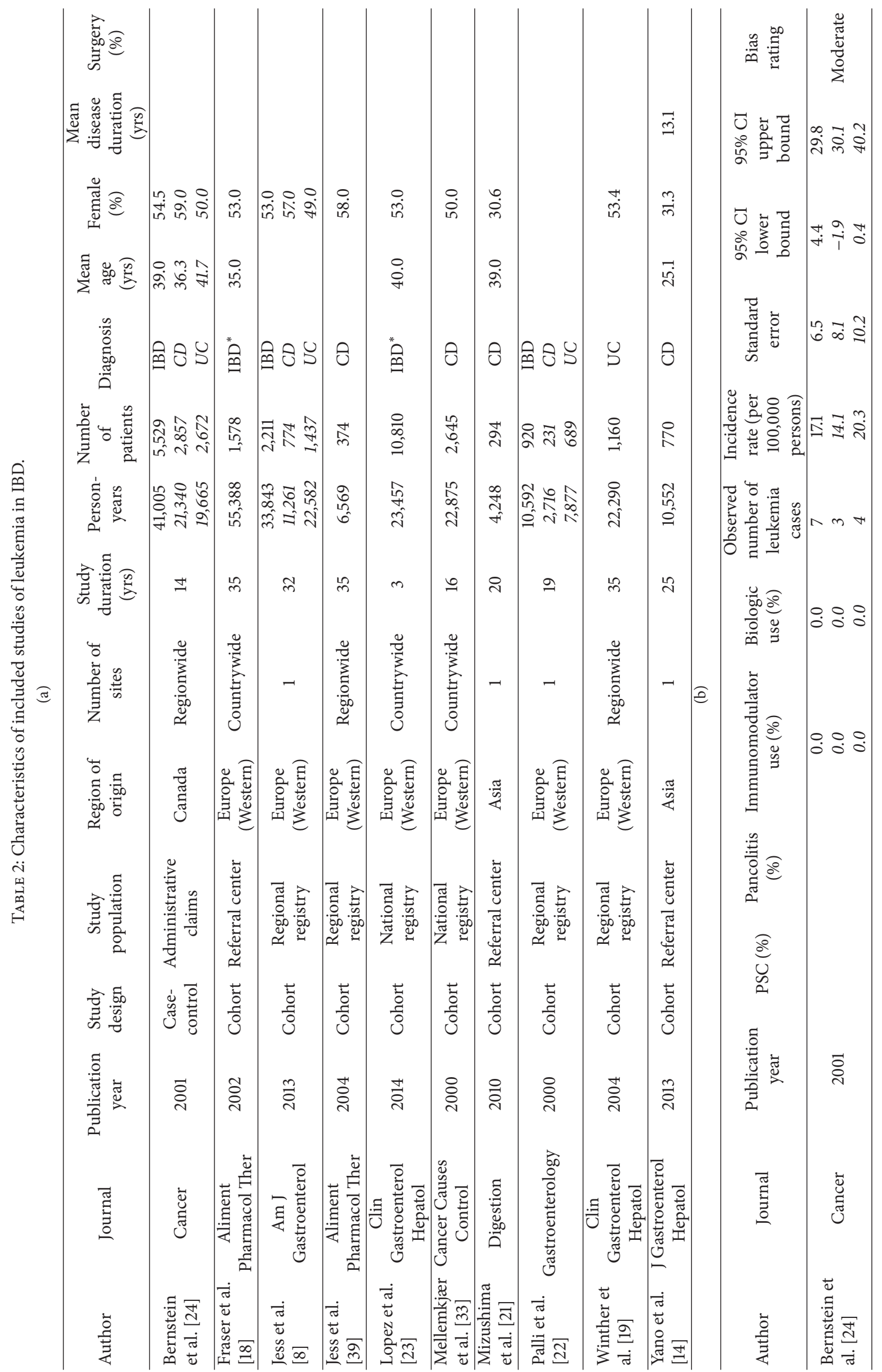




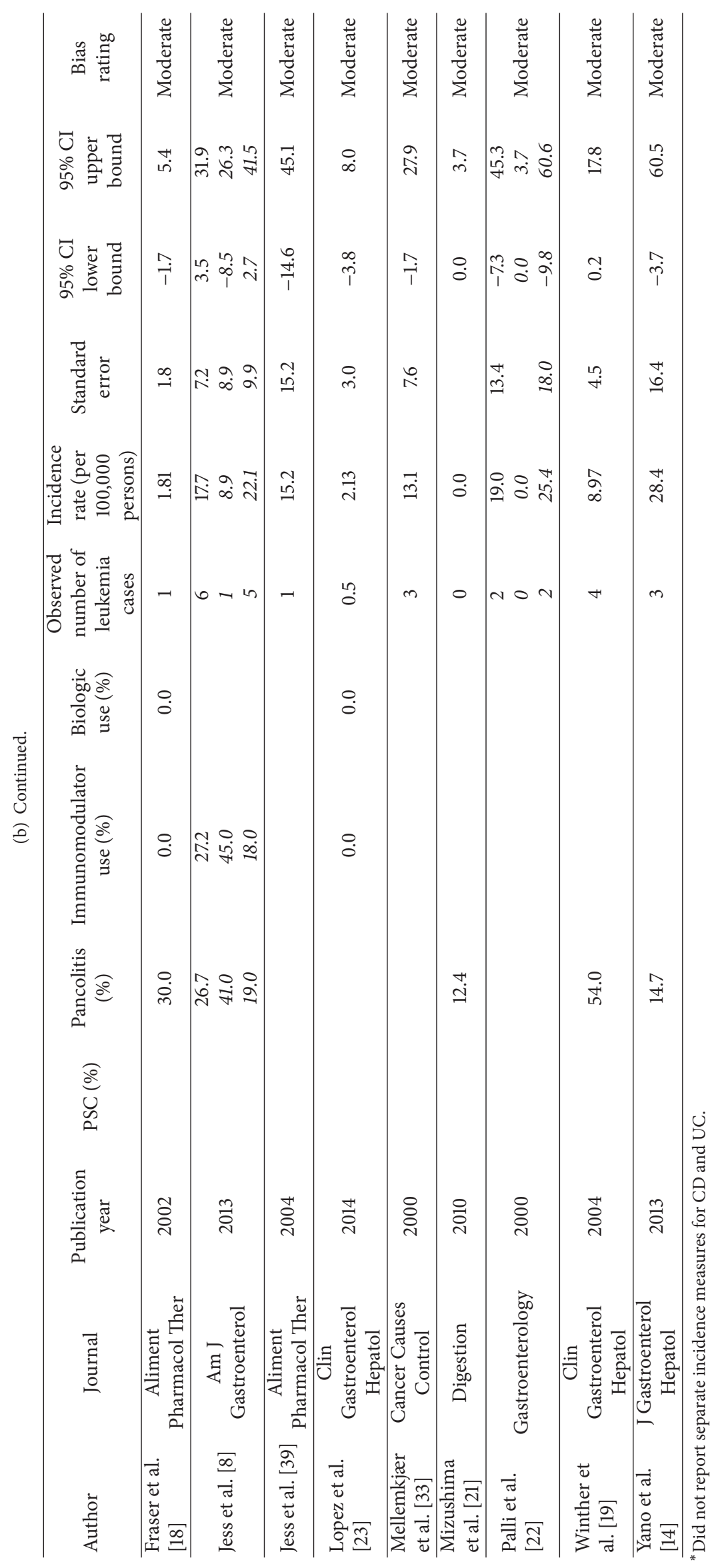




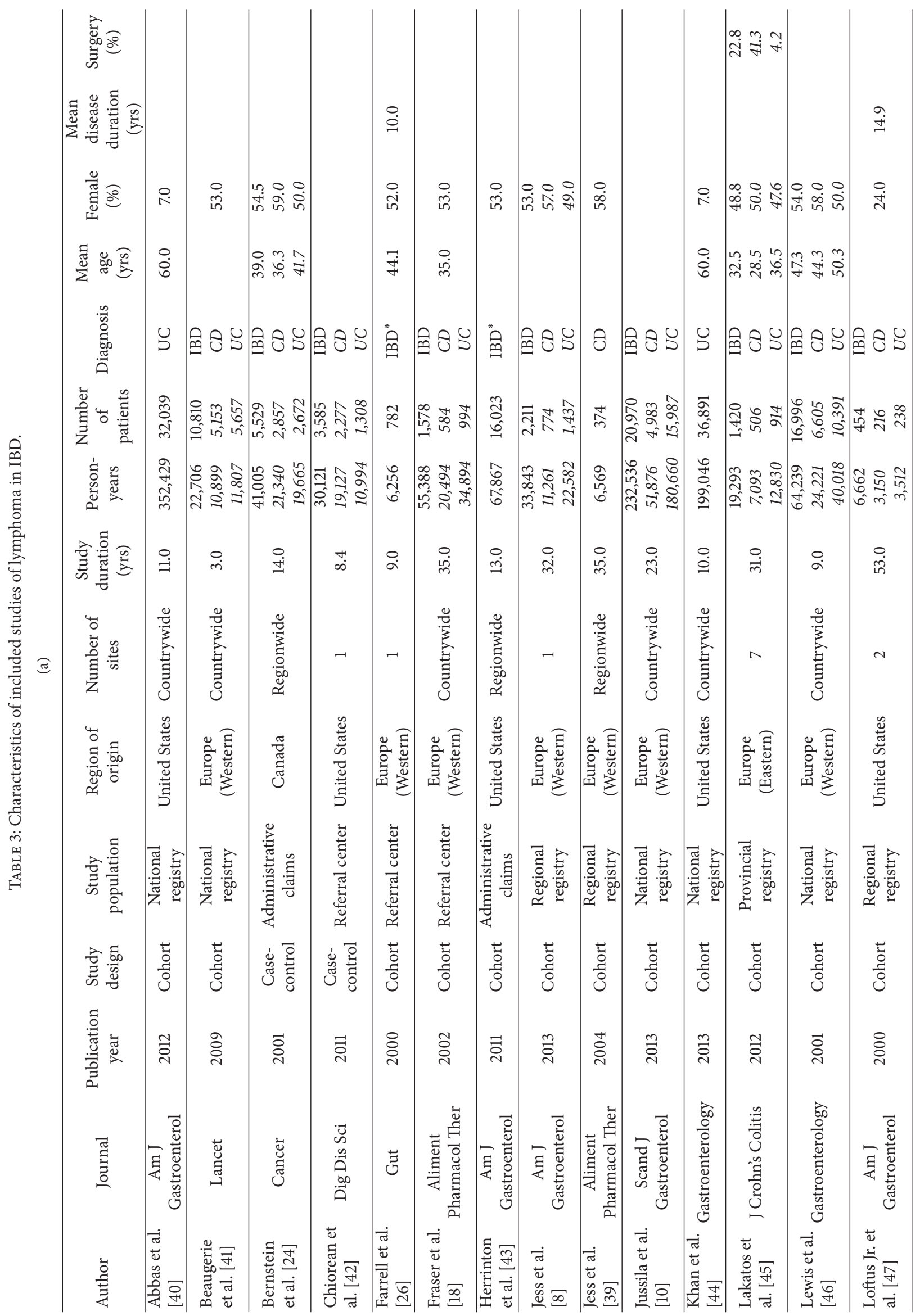




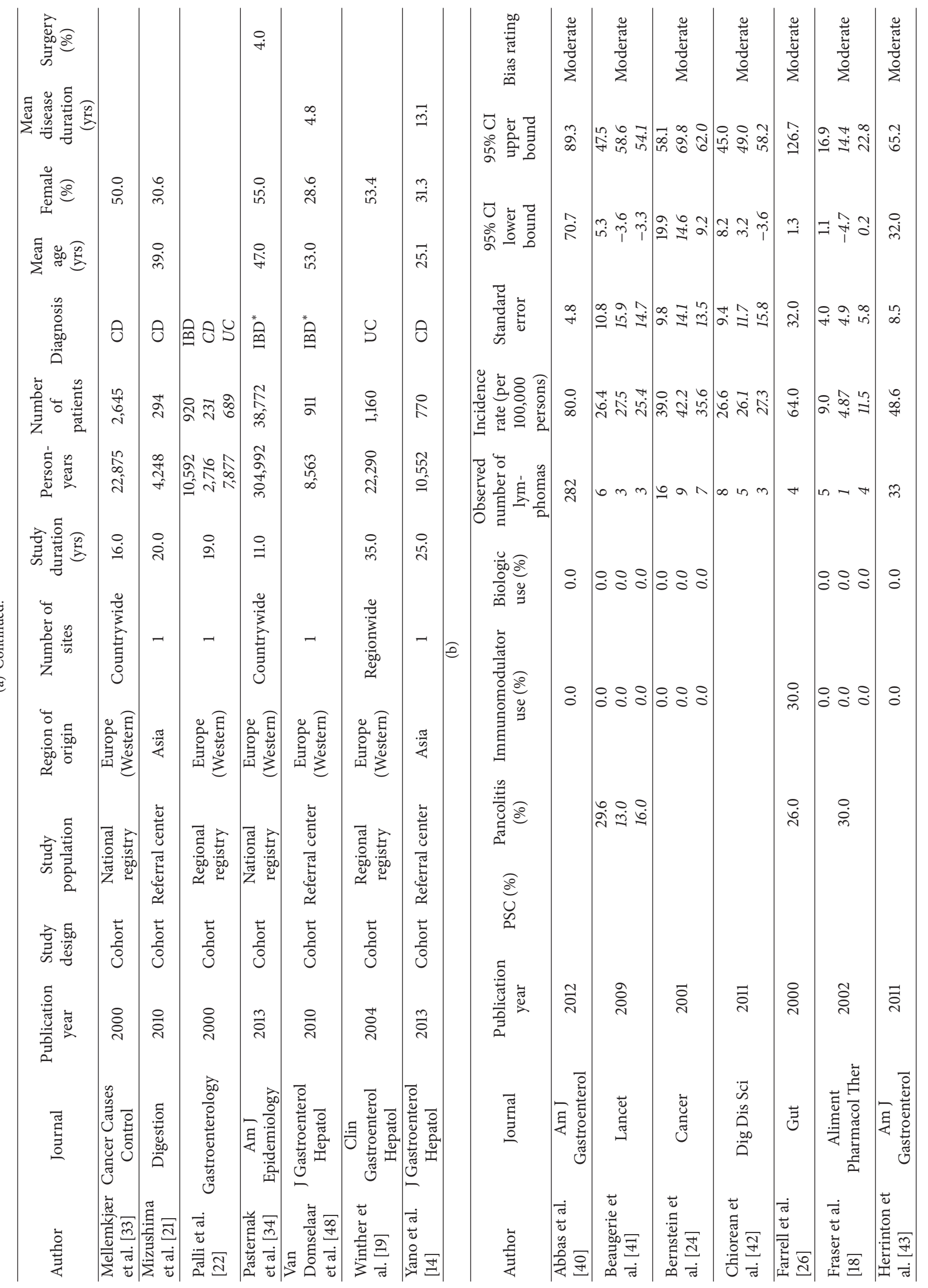




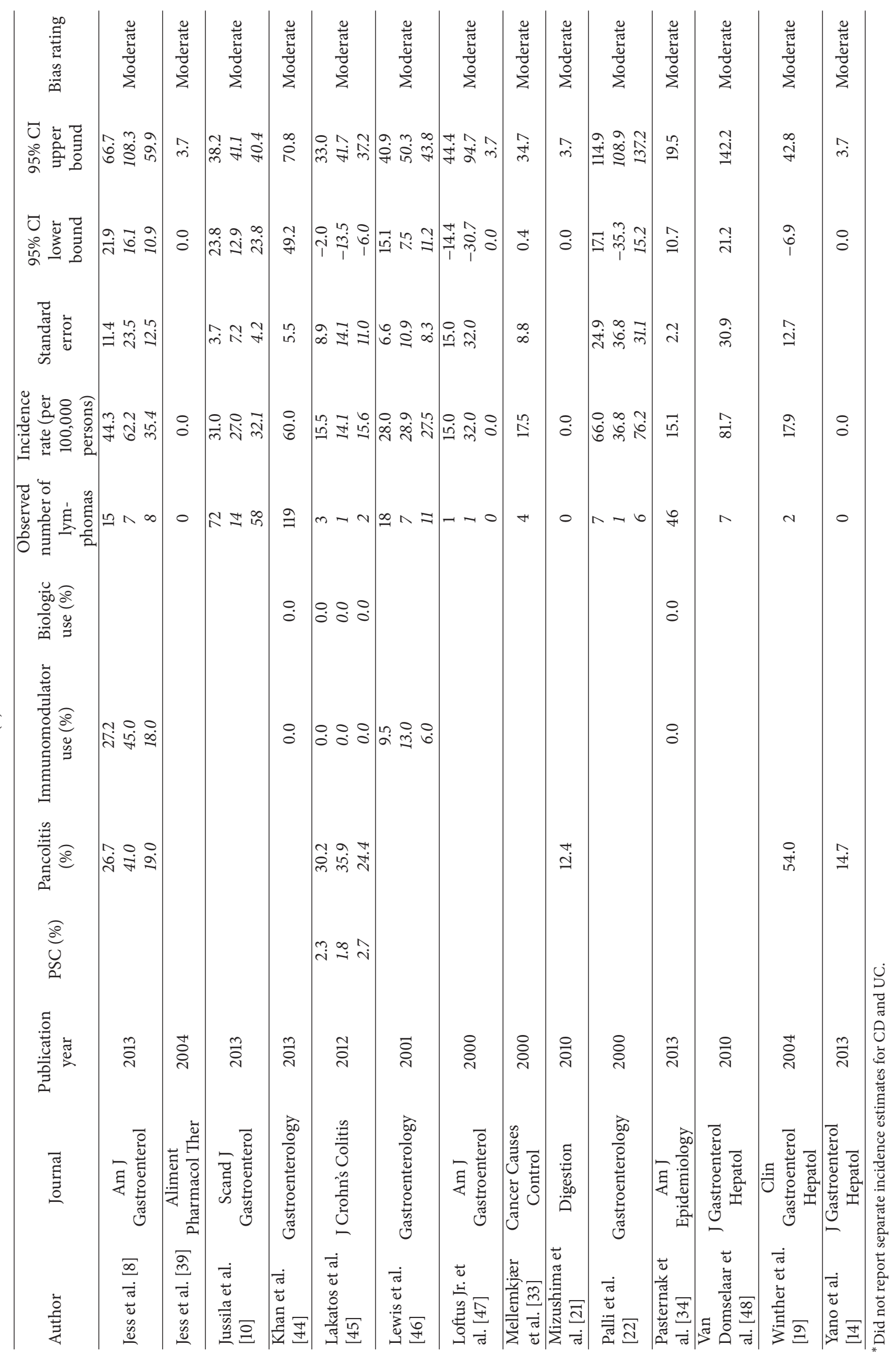




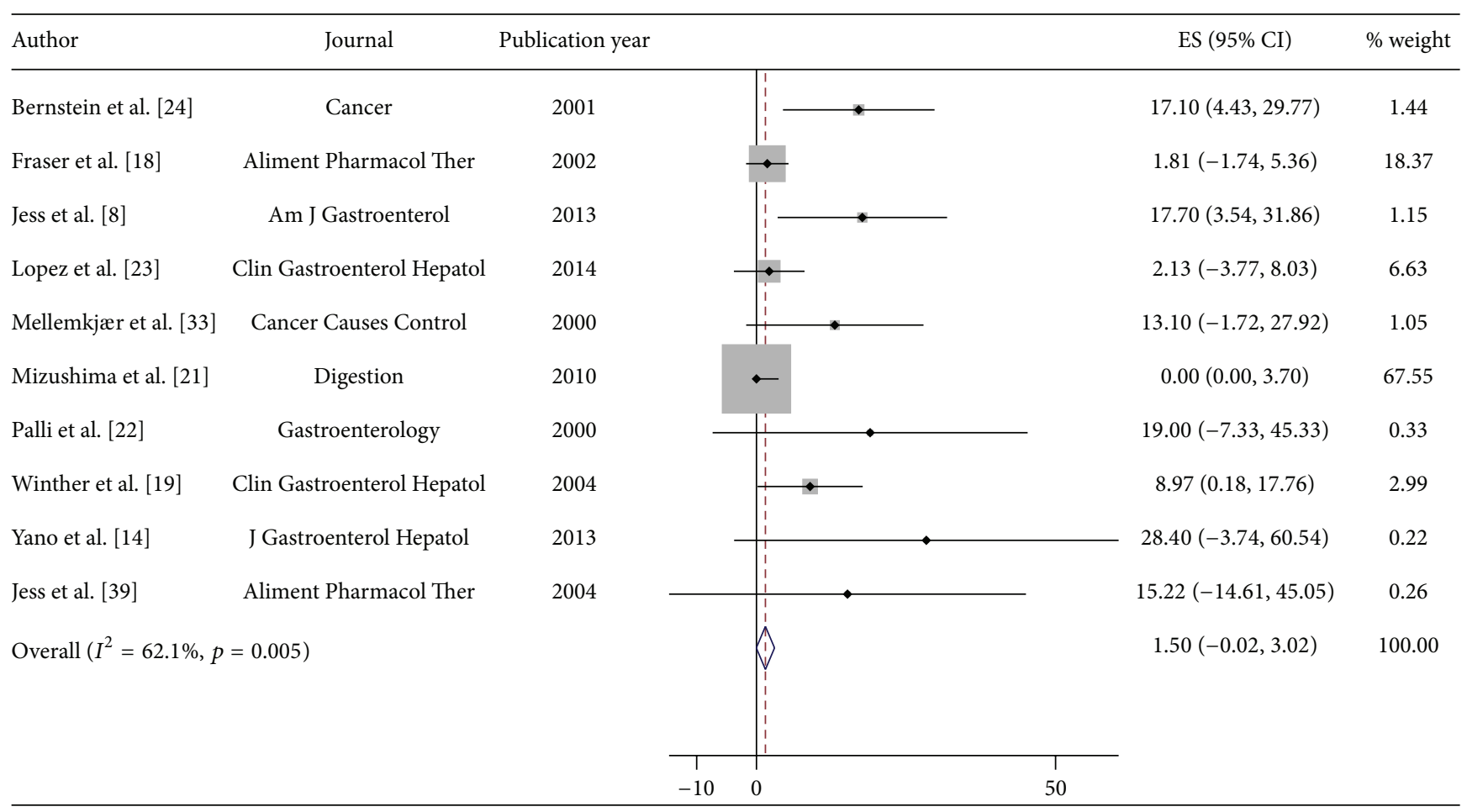

FIGURE 3: Incidence of leukemia in patients with inflammatory bowel disease (IBD). Each incidence estimate is presented followed by the $95 \%$ confidence intervals (CIs). Each square in the plot indicates the point estimate of the incidence. The diamond represents the summary incidence from the pooled studies. Error bars depict the 95\% CIs.

3.2. Discussion. This meta-analysis was performed in order to produce updated and reliable incidence rates for CRC, leukemia, and lymphoma in IBD patients and in CD and UC separately. We aimed to quantify cancer incidence associated with underlying IBD, without the effects of immunomodulator and biologic pharmacotherapy, but this was difficult without reliable reporting of treatment information in the available studies. Although we could not pool estimates of the incidence of CRC in IBD and UC specifically, a pooled incidence rate of $53.3 / 100,000$ py (95\% CI $46.3-$ $60.3 / 100,000)$ in CD was obtained. The estimated worldwide CRC incidence rate is $19.3 / 100,000$ py [53]. In more developed regions of the world, which compares to the regions of origin of the included studies, the incidence rate is higher at 59.2/100,000 py [53]. As such, CRC incidence in CD does not appear to be higher than that of the general population in similar areas of origin. Of note, these incidence estimates are crude (not age-adjusted) and therefore may not reflect differences in the age of the underlying populations.

For leukemia, pooled incidence rates of $1.5 / 100,000$ py (95\% CI -0.06-3.0/100,000), 0.3/100,000 py (95\% CI -1.0$1.6 / 100,000)$, and $13.0 / 100,000$ py (95\% CI 5.8-20.3/100,000) were obtained for IBD, CD, and UC, respectively. The estimated worldwide leukemia incidence is $5.0 / 100,000$ py and $11.3 / 100,000$ py in developed regions [53]. Thus, the incidence of leukemia in IBD and CD is lower than that of the general population in developed regions but is slightly higher in UC. For lymphoma, substantial heterogeneity prevented the pooling of estimates for IBD and UC; however a pooled incidence rate of $0.6 / 100,000$ py $(95 \% \mathrm{CI}-0.4-2.1 / 100,000)$ in $\mathrm{CD}$ was obtained. Estimated worldwide lymphoma incidence is $6.4 / 100,000$ py and $17.6 / 100,000$ py in more developed areas [53]. Thus, the incidence of lymphoma in CD is lower than estimated both worldwide and in developed regions.

Due to incomplete reporting of use of immunomodulators and biologics in the published literature, we could not calculate incidence rates of CRC, leukemia, and lymphoma specifically in persons not treated with these medications; however incidence estimates stratified by publication year before and after widespread use of these medications were not significantly different. This suggests that the impact of immunomodulators and biologics on the incidence of these cancers may be negligible. Metaregression did not reveal any significant subject- or study-level covariate effects in the majority of analyses, with the exception of the effect of mean age on the incidence of lymphoma in IBD. The power of these tests was limited by incomplete reporting of these variables and the small number of included studies.

The strength of the present study is the comprehensiveness of the literature search and evaluation of data for inclusion. Despite the exhaustiveness of the search, we could include only a small number of studies, limiting the power of the pooled analyses and ultimate confidence in incidence estimates. In addition, substantial heterogeneity prevented pooling of estimates in some cases. The heterogeneity of the included studies may reflect differences in follow-up time, cohort size, geographic differences in patient care, or other factors that we were unable to assess due to incomplete 


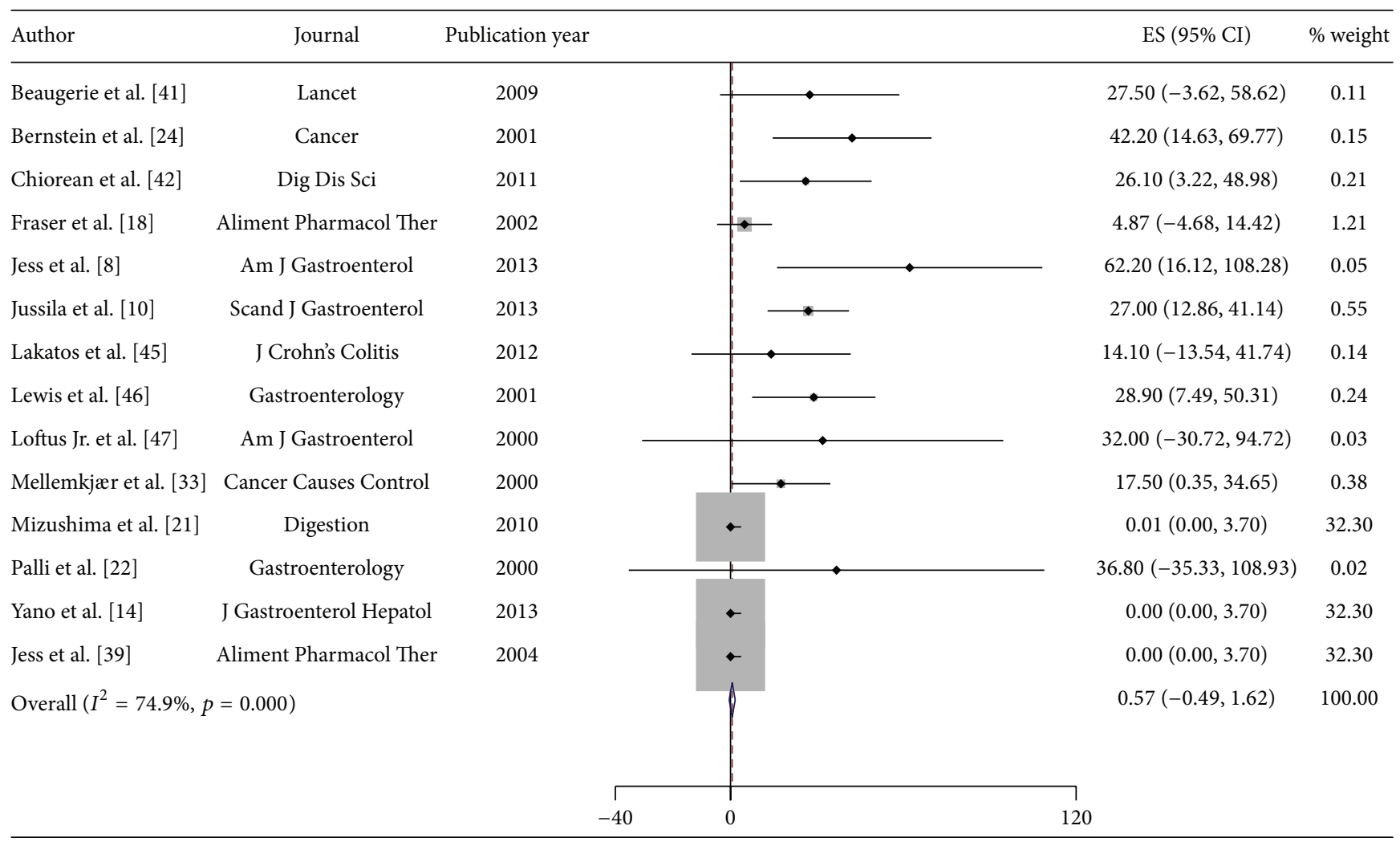

FIGURE 4: Incidence of lymphoma in patients with Crohn's Disease (CD). Each incidence estimate is presented followed by the $95 \%$ confidence intervals (CIs). Each square in the plot indicates the point estimate of the incidence. The diamond represents the summary incidence from the pooled studies. Error bars depict the $95 \%$ CIs.

reporting in the published literature. Although these limitations may lead to bias in our incidence estimates, the direction of which is indeterminable, our estimates are based on the best available evidence.

\section{Conclusions}

This meta-analysis presents updated estimates of the incidence of CRC, leukemia, and lymphoma in adults with IBD. Overall, the incidence of these malignancies does not appear to be higher than in the general population. Further research is needed to explore patient characteristics that may modify the risk of malignancy. Specifically, we need large population based cohort studies in IBD patients that report complete demographic and outcome data. Detailed information on immunomodulator and biologic use is limited in the published literature, and if we are to be able to truly understand the potential increased risk of malignancy associated with IBD pharmacotherapy, this information is required.

\section{Abbreviations}

CRC: Colorectal cancer

IBD: Inflammatory bowel disease

PSC: Primary sclerosing cholangitis

LD: Lymphoproliferative disorders

CD: Crohn's Disease
UC: Ulcerative colitis

CI: Confidence interval

py: Person-years.

\section{Competing Interests}

The authors declare that they have no competing interests.

\section{References}

[1] J. K. Dyson and M. D. Rutter, "Colorectal cancer in inflammatory bowel disease: what is the real magnitude of the risk?" World Journal of Gastroenterology, vol. 18, no. 29, pp. 3839-3848, 2012.

[2] E. R. Kim and D. K. Chang, "Colorectal cancer in inflammatory bowel disease: the risk, pathogenesis, prevention and diagnosis," World Journal of Gastroenterology, vol. 20, no. 29, pp. 9872-9881, 2014.

[3] J. K. Triantafillidis, G. Nasioulas, and P. A. Kosmidis, "Colorectal cancer and inflammatory bowel disease: epidemiology, risk factors, mechanisms of carcinogenesis and prevention strategies," Anticancer Research, vol. 29, no. 7, pp. 2727-2737, 2009.

[4] J. E. Baars, E. J. Kuipers, M. van Haastert, J. J. Nicolaï, A. C. Poen, and C. J. van der Woude, "Age at diagnosis of inflammatory bowel disease influences early development of colorectal cancer 
in inflammatory bowel disease patients: a nationwide, longterm survey," Journal of Gastroenterology, vol. 47, no. 12, pp. 1308-1322, 2012.

[5] V. Bergeron, A. Vienne, H. Sokol et al., "Risk factors for neoplasia in inflammatory bowel disease patients with pancolitis," American Journal of Gastroenterology, vol. 105, no. 11, pp. 24052411, 2010.

[6] F. G. Campos, M. G. Teixeira, A. Scanavini, M. G. D. Almeida, S. C. Nahas, and I. Cecconello, "Intestinal and extraintestinal neoplasia in patients with inflammatory bowel disease in a tertiary care hospital," Arquivos de Gastroenterologia, vol. 50, no. 2, pp. 123-129, 2013.

[7] T. Jess, J. Simonsen, K. T. Jorgensen, B. V. Pedersen, N. M. Nielsen, and M. Frisch, "Decreasing risk of colorectal cancer in patients with inflammatory bowel disease over 30 years," Gastroenterology, vol. 143, no. 2, pp. 375-381, 2012.

[8] T. Jess, E. Horváth-Puhó, J. Fallingborg, H. H. Rasmussen, and B. A. Jacobsen, "Cancer risk in inflammatory bowel disease according to patient phenotype and treatment: a danish population-based cohort study," American Journal of Gastroenterology, vol. 108, no. 12, pp. 1869-1876, 2013.

[9] C. M. Johnson, C. Wei, J. E. Ensor et al., "Meta-analyses of colorectal cancer risk factors," Cancer Causes and Control, vol. 24, no. 6, pp. 1207-1222, 2013.

[10] A. Jussila, L. J. Virta, E. Pukkala, and M. A. Färkkilä, "Malignancies in patients with inflammatory bowel disease: a nationwide register study in Finland," Scandinavian Journal of Gastroenterology, vol. 48, no. 12, pp. 1405-1413, 2013.

[11] P. Manninen, A.-L. Karvonen, H. Huhtala et al., "The risk of colorectal cancer in patients with inflammatory bowel diseases in Finland: a follow-up of 20 years," Journal of Crohn's and Colitis, vol. 7, no. 11, pp. e551-e557, 2013.

[12] M. H. Imam, E. W. Thackeray, and K. D. Lindor, "Colonic neoplasia in young patients with inflammatory bowel disease and primary sclerosing cholangitis," Colorectal Disease, vol. 15, no. 2, pp. 198-203, 2013.

[13] C. P. Selinger, J. M. Andrews, A. Titman et al., "Longterm follow-up reveals low incidence of colorectal cancer, but frequent need for resection, among Australian patients with inflammatory bowel disease," Clinical Gastroenterology and Hepatology, vol. 12, no. 4, pp. 644-650, 2014.

[14] Y. Yano, T. Matsui, F. Hirai et al., "Cancer risk in Japanese Crohn's disease patients: investigation of the standardized incidence ratio," Journal of Gastroenterology and Hepatology, vol. 28, no. 8, pp. 1300-1305, 2013.

[15] F. Magro, L. Peyrin-Biroulet, H. Sokol et al., "Extra-intestinal malignancies in inflammatory bowel disease: results of the 3rd ECCO Pathogenesis Scientific Workshop (III)," Journal of Crohn's and Colitis, vol. 8, no. 1, pp. 31-44, 2014.

[16] M. Mason and C. A. Siegel, "Do inflammatory bowel disease therapies cause cancer?" Inflammatory Bowel Diseases, vol. 19, no. 6, pp. 1306-1321, 2013.

[17] H. Sokol and L. Beaugerie, "Inflammatory bowel disease and lymphoproliferative disorders: the dust is starting to settle," Gut, vol. 58, no. 10, pp. 1427-1436, 2009.

[18] A. G. Fraser, T. R. Orchard, E. M. Robinson, and D. P. Jewell, "Long-term risk of malignancy after treatment of inflammatory bowel disease with azathioprine," Alimentary Pharmacology and Therapeutics, vol. 16, no. 7, pp. 1225-1232, 2002.
[19] K. V. Winther, T. Jess, E. Langholz, P. Munkholm, and V. Binder, "Long-term risk of cancer in ulcerative colitis: a populationbased cohort study from Copenhagen County," Clinical Gastroenterology and Hepatology, vol. 2, no. 12, pp. 1088-1095, 2004.

[20] N. Pedersen, D. Duricova, M. Elkjaer, M. Gamborg, P. Munkholm, and T. Jess, "Risk of extra-intestinal cancer in inflammatory bowel disease: meta-analysis of population-based cohort studies," American Journal of Gastroenterology, vol. 105, no. 7, pp. 1480-1487, 2010.

[21] T. Mizushima, Y. Ohno, K. Nakajima et al., "Malignancy in Crohn's disease: incidence and clinical characteristics in Japan," Digestion, vol. 81, no. 4, pp. 265-270, 2010.

[22] D. Palli, G. Trallori, S. Bagnoli et al., "Hodgkin's disease risk is increased in patients with ulcerative colitis," Gastroenterology, vol. 119, no. 3, pp. 647-653, 2000.

[23] A. Lopez, M. Mounier, A.-M. Bouvier et al., "Increased risk of acute myeloid leukemias and myelodysplastic syndromes in patients who received thiopurine treatment for inflammatory bowel disease," Clinical Gastroenterology and Hepatology, vol. 12, no. 8, pp. 1324-1329, 2014.

[24] C. N. Bernstein, J. F. Blanchard, E. Kliewer, and A. Wajda, "Cancer risk in patients with inflammatory bowel disease: a population-based study," Cancer, vol. 91, no. 4, pp. 854-862, 2001.

[25] J. Askling, P. W. Dickman, P. Karlén et al., "Family history as a risk factor for colorectal cancer in inflammatory bowel disease," Gastroenterology, vol. 120, no. 6, pp. 1356-1362, 2001.

[26] R. J. Farrell, Y. Ang, P. Kileen et al., "Increased incidence of nonHodgkin's lymphoma in inflammatory bowel disease patients on immunosuppressive therapy but overall risk is low," Gut, vol. 47, no. 4, pp. 514-519, 2000.

[27] C. D. Gillen, R. S. Walmsley, P. Prior, H. A. Andrews, and R. N. Allan, "Ulcerative colitis and Crohn's disease: a comparison of the colorectal cancer risk in extensive colitis," Gut, vol. 35, no. 11, pp. 1590-1592, 1994.

[28] L. J. Herrinton, L. Liu, T. R. Levin, J. E. Allison, J. D. Lewis, and F. Velayos, "Incidence and mortality of colorectal adenocarcinoma in persons with inflammatory bowel disease from 1998 to 2010," Gastroenterology, vol. 143, no. 2, pp. 382-389, 2012.

[29] J. K. Hou, J. R. Kramer, P. Richardson, M. Mei, and H. B. ElSerag, "Risk of colorectal cancer among Caucasian and African American veterans with ulcerative colitis," Inflammatory Bowel Diseases, vol. 18, no. 6, pp. 1011-1017, 2012.

[30] P. L. Lakatos, G. David, T. Pandur et al., "Risk of colorectal cancer and small bowel adenocarcinoma in Crohn's disease: a population-based study from western Hungary 1977-2008," Journal of Crohn's and Colitis, vol. 5, no. 2, pp. 122-128, 2011.

[31] J. E. Lennard-Jones, D. M. Melville, B. C. Morson, J. K. Ritchie, and C. B. Williams, "Precancer and cancer in extensive ulcerative colitis: findings among 401 patients over 22 years," Gut, vol. 31, no. 7, pp. 800-806, 1990.

[32] B. D. Lovasz, L. Lakatos, P. A. Golovics et al., "Risk of colorectal cancer in Crohn's disease patients with colonic involvement and stenosing disease in a population-based cohort from Hungary," Journal of Gastrointestinal and Liver Diseases, vol. 22, no. 3, pp. 265-268, 2013.

[33] L. Mellemkjær, C. Johansen, G. Gridley, M. S. Linet, S. K. Kjær, and J. H. Olsen, "Crohn's disease and cancer risk (Denmark)," Cancer Causes and Control, vol. 11, no. 2, pp. 145-150, 2000. 
[34] B. Pasternak, H. Svanström, K. Schmiegelow, T. Jess, and A. Hviid, "Use of azathioprine and the risk of cancer in inflammatory bowel disease," American Journal of Epidemiology, vol. 177, no. 11, pp. 1296-1305, 2013.

[35] F. D. M. van Schaik, M. G. H. van Oijen, H. M. Smeets, G. J. M. G. van der Heijden, P. D. Siersema, and B. Oldenburg, "Thiopurines prevent advanced colorectal neoplasia in patients with inflammatory bowel disease," Gut, vol. 61, no. 2, pp. 235240, 2012.

[36] S. Venkataraman, V. Mohan, B. S. Ramakrishna et al., "Risk of colorectal cancer in ulcerative colitis in India," Journal of Gastroenterology and Hepatology (Australia), vol. 20, no. 5, pp. 705-709, 2005.

[37] E. P. Wandall, P. Damkier, F. Møller Pedersen, B. Wilson, and O. B. Schaffalitzky De Muckadell, "Survival and incidence of colorectal cancer in patients with ulcerative colitis in funen county diagnosed between 1973 and 1993," Scandinavian Journal of Gastroenterology, vol. 35, no. 3, pp. 312-317, 2000.

[38] L. Lakatos, G. Mester, Z. Erdelyi et al., "Risk factors for ulcerative colitis-associated colorectal cancer in a Hungarian cohort of patients with ulcerative colitis: results of a populationbased study," Inflammatory Bowel Diseases, vol. 12, no. 3, pp. 205-211, 2006.

[39] T. Jess, K. V. Winther, P. Munkholm, E. Langholz, and V. Binder, "Intestinal and extra-intestinal cancer in Crohn's disease: follow-up of a population-based cohort in Copenhagen County, Denmark," Alimentary Pharmacology and Therapeutics, vol. 19, no. 3, pp. 287-293, 2004.

[40] A. Abbas, Y. Koleva, and N. Khan, "Lymphoproliferative disorders in patients receiving thiopurines for ulcerative colitis: a nationwide 10 -yearretrospective cohort from the veterans affairs healthcare system," The American Journal of Gastroenterology, vol. 107, article S693, 2012.

[41] L. Beaugerie, N. Brousse, A. M. Bouvier et al., "Lymphoproliferative disorders in patients receiving thiopurines for inflammatory bowel disease: a prospective observational cohort study," The Lancet, vol. 374, no. 9701, pp. 1617-1625, 2009.

[42] M. V. Chiorean, B. Pokhrel, J. Adabala, D. J. Helper, C. S. Johnson, and B. Juliar, "Incidence and risk factors for lymphoma in a single-center inflammatory bowel disease population," Digestive Diseases and Sciences, vol. 56, no. 5, pp. 1489-1495, 2011.

[43] L. J. Herrinton, L. Liu, X. Weng, J. D. Lewis, S. Hutfless, and J. E. Allison, "Role of thiopurine and anti-TNF therapy in lymphoma in inflammatory bowel disease," American Journal of Gastroenterology, vol. 106, no. 12, pp. 2146-2153, 2011.

[44] N. Khan, A. M. Abbas, G. R. Lichtenstein, E. V. Loftus Jr., and L. A. Bazzano, "Risk of lymphoma in patients with ulcerative colitis treated with thiopurines: a nationwide retrospective cohort study," Gastroenterology, vol. 145, no. 5, pp. 1007.e31015.e3, 2013.

[45] L. Lakatos, P. A. Golovics, B. D. Lovasz et al., "Sa1247 Low risk of lymphoma in inflammatory bowel diseases in Western Hungary. Results from a population-based incident cohort," Gastroenterology, vol. 142, no. 5, supplement 1, p. S-253, 2012.

[46] J. D. Lewis, W. B. Bilker, C. Brensinger, J. J. Deren, D. J. Vaughn, and B. L. Strom, "Inflammatory bowel disease is not associated with an increased risk of lymphoma," Gastroenterology, vol. 121, no. 5, pp. 1080-1087, 2001.

[47] E. V. Loftus Jr., W. J. Tremaine, T. M. Habermann, W. S. Harmsen, A. R. Zinsmeister, and W. J. Sandborn, "Risk of lymphoma in inflammatory bowel disease," The American Journal of Gastroenterology, vol. 95, no. 9, pp. 2308-2312, 2000.

[48] M. Van Domselaar, A. López San Román, M. Bastos Oreiro, and E. Garrido Gómez, "Lymphoproliferative disorders in an inflammatory bowel disease unit," Gastroenterologia y Hepatologia, vol. 33, no. 1, pp. 12-16, 2010.

[49] J. Sterne, J. Higgins, and B. Reeves, A Cochrane Risk of Bias Assessment Tool: For Non-Randomized Studies of Interventions (ACROBAT-NRSI), Version 1.0.0, 2014.

[50] J. P. T. Higgins and S. Green, Cochrane Handbook for Systematic Reviews of Interventions, John Wiley \& Sons, 2011.

[51] J. Higgins, J. Deeks, and D. Altman, Special Topics in Statistics, The Cochrane Book, chapter 16, John Wiley \& Sons, London, UK, 2008.

[52] T. M. Palmer and J. A. C. Sterne, Eds., Meta-Analysis in Stata: An Updated Collection from the Stata Journal, Stata Press, Station, Tex, USA, 2009.

[53] J. Ferlay, I. Soerjomataram, M. Ervik et al., "GLOBOCAN 2012 v1.0, cancer incidence and mortality worldwide," IARC Cancer Base 11, International Agency for Research on Cancer, Lyon, France, 2013. 


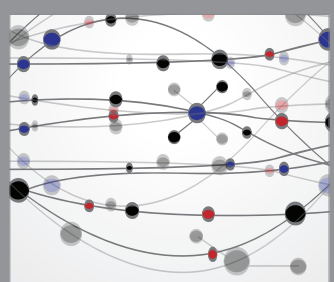

The Scientific World Journal
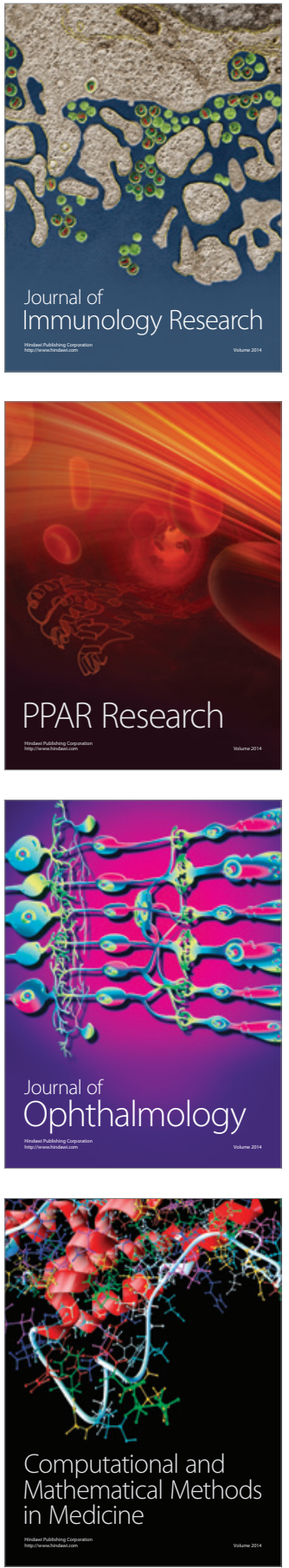

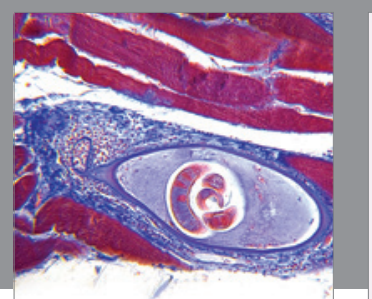

Gastroenterology Research and Practice

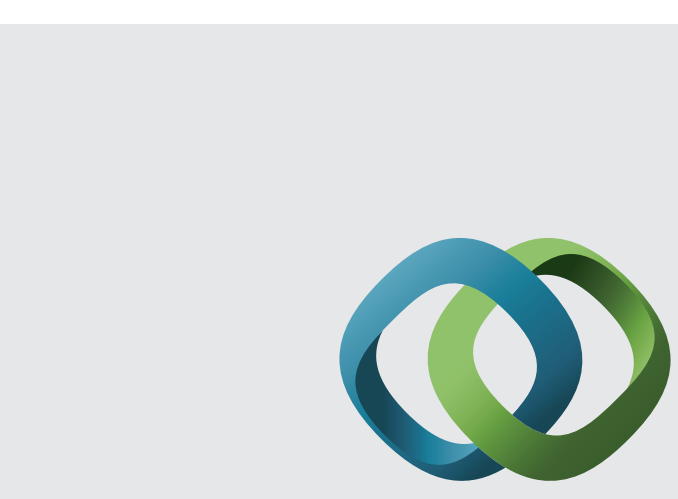

\section{Hindawi}

Submit your manuscripts at

http://www.hindawi.com
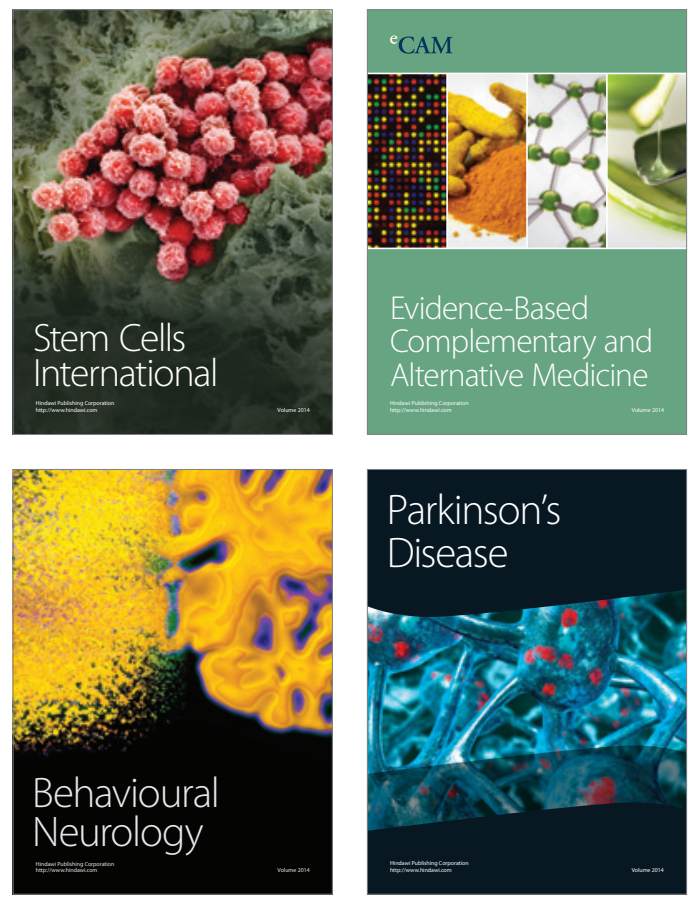
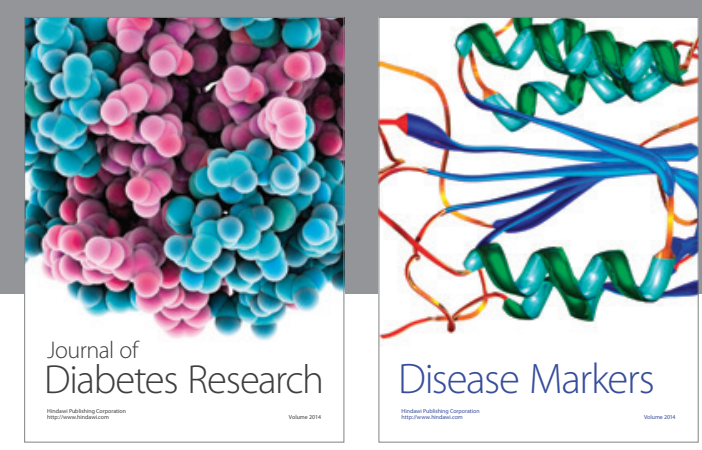

Disease Markers
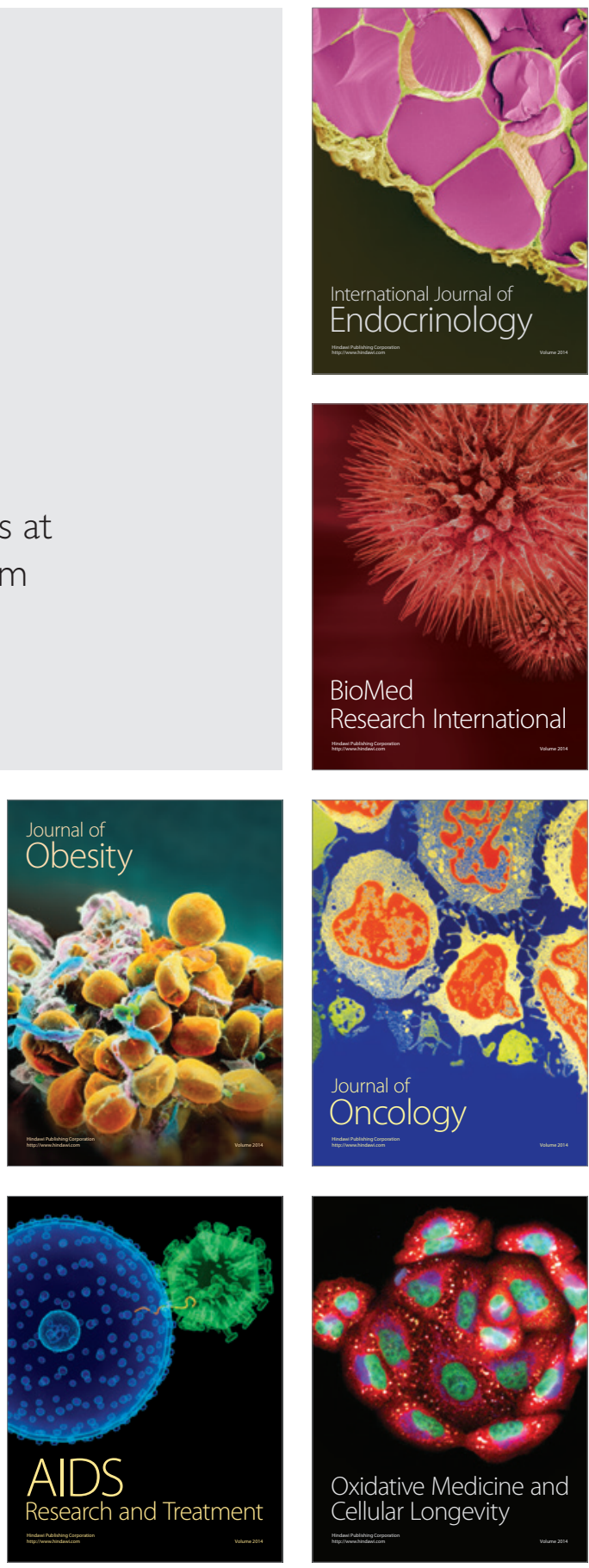